\title{
Ultrasound features of a metastatic seminal vesicle melanoma: A case report
}

\author{
Andrea Fabiani ${ }^{1}$, Emanuele Principi ${ }^{2}$, Alessandra Filosa ${ }^{3}$, Fabrizio Fioretti ${ }^{1}$, Valentina Maurelli ${ }^{1}$, \\ Lucilla Servi $^{1}$, Gabriele Mammana ${ }^{4}$ \\ ${ }^{1}$ Urology Unit, Surgery Department, Macerata Civic Hospital, Area Vasta 3 ASUR Marche, Italy; \\ ${ }^{2}$ Urologic Clinic, Polytechnic University of Marche Region, Ancona, Italy; \\ ${ }^{3}$ Pathologic Unit ASUR Marche Area Vasta 3, Macerata Civic Hospital, Italy.
}

\begin{abstract}
Summary In this report we describe what we consider to be the second case of seminal vescicle (SV) metastasis from an unknown primary melanoma. only presenting symptom was a palpable firm nodule of the right prostate base on digital rectal examination (DRE).

The diagnosis, after prostatic transrectal ultrasound examination (TRUS), was performed by ultrasound guided biopsy.

We underline that prostatic TRUS evaluation is mandatory in case of abnormal digital rectal examination. Seminal vesicle must be always evaluated.
\end{abstract}

KEY WORDS: Metastatic melanoma;

Seminal vesicle; Transrectal ultrasound; CUP-syndrome.

Submitted 15 November 2016; Accepted 15 December 2016

\section{INTRODUCTION}

Metastatic melanoma of the seminal vesicles is extremely rare (1). Only one case has been reported of metastatic malignant melanoma to the male genital organs, in patients with an unknown primary melanoma (2). In other two cases reports a metastasis to the seminal vesicle from a primary cutaneous malignant melanoma has been described (3). We report the second case of seminal vesicle (SV) metastasis from an unknown primary melanoma.

\section{Case presentation}

A 78-years-old man presented for an urologic screening during the follow up of a gastric carcinoma, surgically resected some years ago. His serum prostate-specific antigen level was 2,06 ng/ml. International Prostatic Index Score was 6. At digital rectal examination, the prostate resulted modestly increased in volume with a firm nodule palpable at the right base. Transrectal ultrasound (TRUS) examination, performed with a 6-12 Mhz ultrasound end fire probe (8818 BK Medical, Denmark), revealed an hypo-echoic inhomogeneous nodular lesion of $3 \mathrm{~cm}$ in major diameter, occupying the site of seminal vesicle normal location (Figure 1). Ecocolor signal was present (Figure 2). Transrectal ultrasound guided prostate biopsy was performed. Pathology revealed seminal vesicle invasion by a malignant tumor with S-100 and MMB45 expression on immunohistochemical examination, suggesting a melanoma metastasis (Figure 3a, b, c). Further extension assessment by cistoscopy, wholebody computed tomography, dermatologic, ophthalmologic and gastroenteric examinations showed no primary tumor. Since he had a single localization, surgical resection was proposed.

\section{Figure 1.}

Inhomogeneous nodular lesion of $3 \mathrm{~cm}$ in major diameter, occupying the site of right seminal vesicle normal location. The aspect is similar to that of advanced prostate cancer.

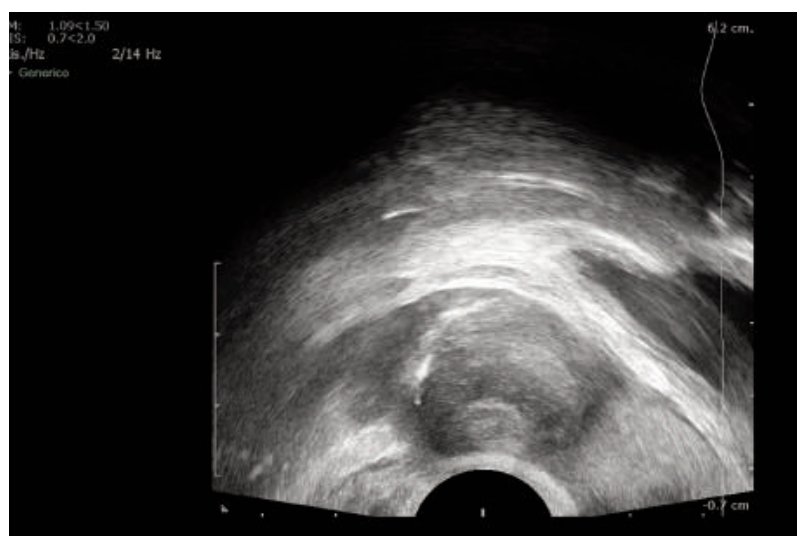

Figure 2.

Echocolor signal was present in the retro- vesical solid mass.

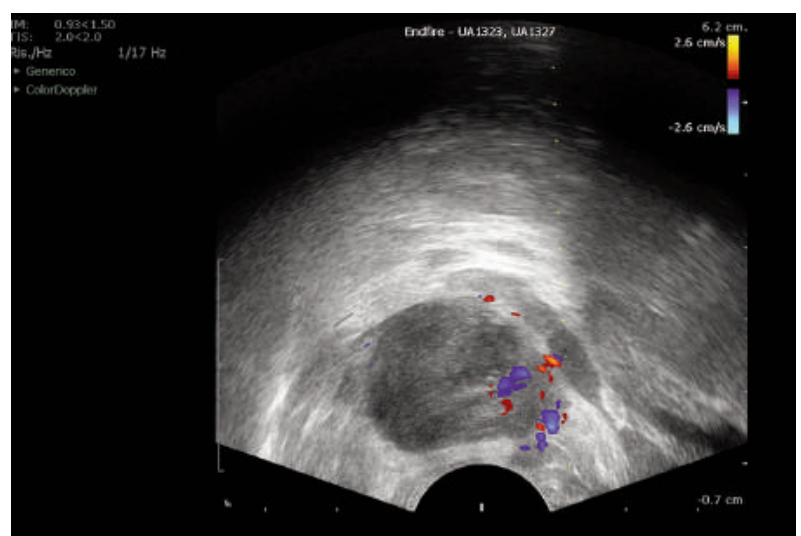




\section{Figure 3.}

The microscopic features of transrectal bioptic core obtained from the solid lesion; three different grade of magnifications.
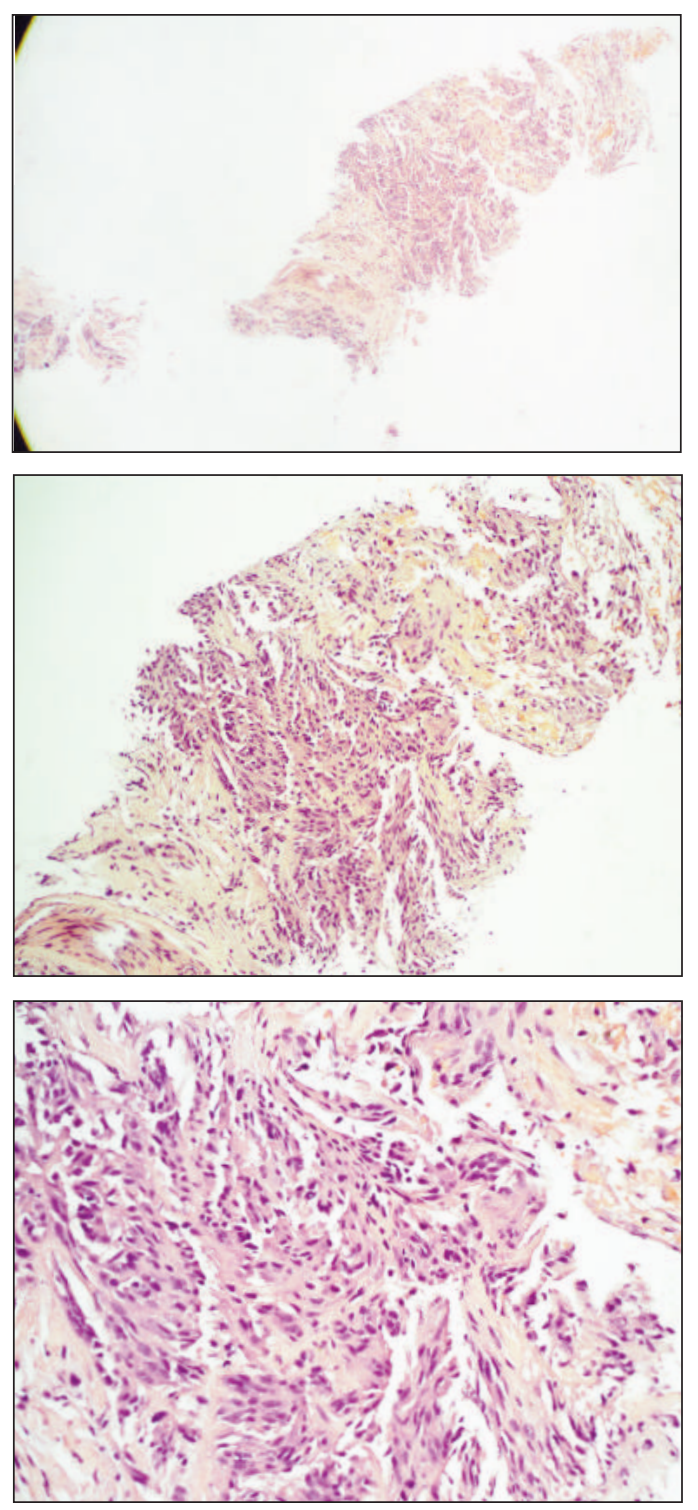

C.

The patient denied any further treatment. He was lost at 6 months visit follow-up.

\section{Discussion}

Metastatic solid tumor in the male genital urinary tract are under diagnosed. They are considered uncommon but an accurate diagnosis is important because of its aggressiveness and possible functional impact (1). Clinical manifestations are usually lower urinary tract symptoms, haematuria, pelvic pain, haemospermia, melanospermia. In our case, the absence of sexual activity by the patient has not allowed the identification of the lesion before digital rectal exploration (DRE) was performed. In case of DRE abnormality, TRUS is the initial diagnostic tool of choice. Performing a TRUS, it must be taken into account that the prostate must be analyzed on two orthogonal planes, transverse and longitudinal. In this study it is essential to examine the juxtavesical ureters, bladder, prostate and seminal vesicles (4). In cases of suspicious masses of the SV, TRUS allows an excellent visualization. At the same time it offers the opportunity of performing a TRUS-guided biopsy for histological confirmation (5). Additional radiological evaluation with magnetic resonance imaging and computed tomography is required to visualize the exact extent of metastases and concomitant pelvic pathology (6). Normally, at TRUS evaluation, SV are seen as elongated septate cystic structures above the prostate. The distal portion of the vas deferens (VD) is seen as a slightly dilated tubular structure (the ampulla of the VD) medial to the SV. If scanned obliquely, the SV and the terminal portion of the VD can be seen joining to form the ejaculatory duct (ED), which may be traced to the region of the veru montanum (7). Ultrasound features in case of SV diseases are not specific. Primary neoplasms are extremely rare. They are classically classified as benign or malignant, primary or secondary. Benign tumors of the SV are even rarer and the reported histology types in the literature are cystic dysplasia, fibroepithelial tumor, leiomyoma, cystadenoma and Schwannoma (8).

Primary adenocarcinoma is the most common neoplasm of the SV. Other histology types of the primary neoplasms of the SV that have been recorded in the literature are sarcomas, squamous cell carcinoma, yolk salk tumor, neuroendocrine carcinoma, paraganglioma, epithelial stromal tumors, lymphoma (Burkitt, B-Cell), extragastrointestinal stromal tumor (EGIST), myxoid solitary fibrous tumor, and seminoma peripheral primitive neuroectodermal tumor (PPNET) (9).

Secondary SV involvement is most frequent from the prostate but other tumors of adjacent organs such as the bladder and rectum, as well as metastasis from distant organs or peritoneal carcinomatosis, may also affect the SV (10). These tumors may appear as a retrovesical mass with or without prostatic or ureteral obstruction or as an infiltrating lesion in the SV with enhancement similar to that of advanced prostate cancer (7). Also in our reported case, the ultrasound findings were not specific. Only hystolopathological evaluation on biopsy cores obtained by TRUS, associated to ancillary immunohistochemical procedures (S-100 and MMB45) allowed the diagnosis. The primary melanoma was not recognized. We are in presence of a so called CUP-syndrome that comprises a heterogenous group of metastatic tumors for which no primary site can be detected. Although most CUPSyndromes derive from neuroendocrine carcinomas, also metastatic melanomas are sporadically detected by metastasis only $(11,12)$.

Performing a brief literature review using the term "seminal vesicle melanoma", we found only three cases reported. In two cases $(3,13)$, primary melanoma was identified. In one case (2), as reported in our experience, the initial localization of the melanoma remained unknown. Anyway, genito-urinary locations of metastatic malignant melanoma have a poor prognosis. Surgical excision with no margin should always be discussed as first-line management whenever possible, with or without systemic therapy. 


\section{Conclusion}

Diseases involving the SV are rare. Owing to their close proximity to the prostate gland, these organs are most commonly affected by disease arising from the prostate. Secondary tumor invasion is often seen in patients with prostate, bladder, or rectal cancer. Rarely, primary neoplasms can also occur. Metastatic solid tumor involvement of SV is probably under-diagnosed. Transrectal ultrasound prostatic evaluation is mandatory in case of abnormal digital rectal examination. Seminal vesicle must be always evaluated. At our knowledge, this is the second case of metastatic malignant melanoma of the seminal vescicle in a patient with an unknown primary melanoma.

\section{References}

1. Morichetti D, Mazzucchelli R, Lopez-Beltran A, et al. Secondary neoplasms of the urinary system and male genital organs. BJU Int. 2009; 104:770-6.

2. Meng MV, Werboff LH. Hematospermia as the presenting symptom of metastatic malignant melanoma of unknown primary origin. Urology. 2000; 56:330.

3. Foahom Kamwa AD, Mateus C, Thanigasalam R, et al. Seminal vesicle metastasis of cutaneous malignant melanoma: An unusual and challenging presentation. Can Urol Assoc J. 2015; 9:E220-3.

4. Martino P. Galosi AB, Bitelli $M$, et al. Practical recommendations for performing ultrasound scanning in the urological and andrological fields. Arch Ital Urol Androl: 2014; 86:56-88.
5. Zhang K, Li SQ, He ZJ, et al. Long term efficacy of TRUS-giuded transperineal needle aspiration and irrigation on persistent hematospermia. Zhonghua Nan Ke Xue. 2005; 11:452.

6. Cho I, Lee M, Rha K, et al. Magnetic resonance imaging in hemospermia. J Urol. 1997; 157:258.

7. Kim B, Kawashima A, Ryu JA, et al. Imaging of the seminal vesicle and the vas deferens. RadioGraphics 2009; 29:1105-1121.

8. Sandlow JI, Winfield HN, Goldstein M. Surgery of the scrotum and seminal vesicles. In: Wein AJ, Kavoussi LR, Novick AC, Partin AW, Peters CA, eds. Campbell-Walsh urology. 9th ed, vol 1. Philadelphia, Pa: Saunders Elsevier. 2007; 1098-1127.

9. Katafigiotis I, Sfoungaristos S, Duvdevani M. Primary adenocarcinoma of the seminal vesicles. A review of the literature. Arch Ital Urol Androl. 2016; 88:47-51.

10. King BF, Hattery RR, Lieber MM, Williams B Jr, et al. Seminal vesicle imaging. RadioGraphics. 1989; 9:653-676.

11. Pavlidis N, Briasoulis E, Hainsworth J, et al. Diagnostic and therapeutic management of cancer of unknown primary. Eur $J$ Cancer. 2003; 39:1990.

12. Anbari KK, Schuchter LM, Bucky LP, et al. Melanoma of unknown primary site, presentation treatment and prognosis- a single institution study. Cancer. 1997; 79:1816.

13. Papoutsoglou N, Burger M, Riedmiller H. Persistent painless hemospermia due to metastatic melanoma of the right seminal vesicle BMC Urology. 2013; 13:43.

\section{Correspondence}

Andrea Fabiani, MD

andreadocl@libero.it

Fabrizio Fioretti, MD PhD

fa.fioretti@libero.it

Valentina Maurelli, MD

valentinamaurelli@hotmail.it

Lucilla Servi, MD

lucilla.servi@sanita.marche.it

Surgery Dpt, Section of Urology

ASUR Marche Area Vasta 3 Macerata Hospital, Italy

Emanuele Principi, MD, Urology Resident

principie@tiscali.it

Urologic Clinic, Polytechnic University of Marche Region, Ancona, Italy

Alessandra Filosa, MD PhD

alessandrafilosa@yahoo.it

Gabriele Mammana, MD

gabriele.mammana@sanita.marche.it

Head Surgery Dpt, Chief of Section of Urology

ASUR Marche Area Vasta 3, Macerata Hospital, Italy 